Original Research Article

\title{
Histological evaluation of nephroprotective effect of Solanum nigrum fruit extract against gentamicin induced nephrotoxicity in experimental rats
}

\author{
Vimlesh Kushwaha*
}

Department of Pharmacology, G. C. R. G. Institute of Medical Sciences, Lucknow, Uttar Pradesh, India

Received: 01 September 2019 Revised: 12 September 2019 Accepted: 13 September 2019

\section{*Correspondence to:}

Dr. Vimlesh Kushwaha, Email: drvijv2008@gmail.com

Copyright: () the author(s), publisher and licensee Medip Academy. This is an openaccess article distributed under the terms of the Creative Commons Attribution NonCommercial License, which permits unrestricted noncommercial use, distribution, and reproduction in any medium, provided the original work is properly cited.

\begin{abstract}
Background: Drug induced nephrotoxicity, one of the most common renal problem, is a challenge to deal with especially in patients with renal dysfunction. It is responsible for $20 \%$ cases of acute renal failure in the community. Modern medicines are costly and have minimal nephroprotection. Solanum nigrum fruit extract, a cheaper drug, have antioxidant property and may help in nephroprotection.

Methods: Total 54 rats were randomised in 3 groups named G10, G20 and G30 according to 10,20 and 30 days of treatment. In each groups, rats were randomly assigned to any of the three subgroups i.e., control $\mathrm{C}$ group [received normal saline (2 $\mathrm{ml} / 100 \mathrm{gm} /$ day) orally consecutively for test duration], gentamicin treated (GT) group [received normal saline $(2 \mathrm{ml} / 100 \mathrm{gm} /$ day $)$ orally consecutively for test duration and intraperitoneal gentamicin $(40 \mathrm{mg} / \mathrm{kg})$ once daily for last five days] and $S$. nigrum treated (SNT) group [received S. nigrum orally $(200 \mathrm{mg} / \mathrm{kg} /$ day) for the test duration and intraperitoneal gentamicin (40 $\mathrm{mg} / \mathrm{kg}$ ) once daily for last five days]. Rats were sacrificed 24 hours after the last dose of gentamicin injection (on 11th, 21st and 31st day). Excised kidneys were weighted and prepared for histological examination.

Results: The mean weight of kidneys in GT group was significantly higher than the SNT group in all test durations suggestive of decrease in inflammation in SNT group. This was also reflected histologically as SNT group kidney showed less amount of tubular destruction as compared to GT group.

Conclusions: $S$. nigrum extract provide nephroprotection against gentamicin induced nephrotoxicity.
\end{abstract}

Keywords: Nephroprotection, Gentamicin, Solanum nigrum, Proximal convoluted tubule

\section{INTRODUCTION}

The kidney is an essential excretory organ of our body. It conserves essential products depending on the needs of the body. ${ }^{1}$ It is the prime target of several drugs, toxic xenobiotics or chemicals due to more vascularity (20$25 \%$ of cardiac output) and presence of cellular transport systems that cause accumulation of these compounds within the epithelial cells of nephron. Metabolites of the drugs that are excreted from kidney may also cause cellular damage leading to kidney dysfunction. ${ }^{2}$
Renal diseases are among the commonest cause of hospitalization in most of the countries. The yearly incidence of end stage renal disease in India is approximately $150-200$ per million population according to the Indian Chronic Kidney Disease Registry. ${ }^{3,4}$ Drug induced nephrotoxicity, one of the most common renal problems, is an important concern especially in patients with impaired renal functions. It causes approximately $20 \%$ of community and hospital-acquired episodes of acute renal failure. 
Aminoglycoside antibiotics have been widely used for gram-negative bacterial infections. It is one of the most common causes of acute kidney disease. Gentamicin nephrotoxicity occurs in about $15-30 \%$ of treated subjects. It manifests clinically as non-oliguric renal failure, with a slow rise in serum creatinine and hypoosmolar urinary output developing after several days of treatment. ${ }^{6}$ Gentamicin induced nephrotoxicity is characterized by direct tubular necrosis, without morphological changes in glomerular structures. ${ }^{7}$

Modern medicines are costly and lack reliable nephroprotective action. In recent years, great efforts have been focused on traditional and herbal medicine for the treatment of ARF. ${ }^{8}$ Global estimates show that $80 \%$ of world population cannot afford synthetic pharmaceutical products, hence depend upon traditional medicines that are commonly derived from plants. ${ }^{9}$

Solanum nigrum (Black Nightshade or Black Makoi) is a medicinal plant member of the Solanaceae family of plants. S. nigrum has been used traditionally to treat various ailments such as pain, inflammation, fever and enteric diseases. ${ }^{10,11}$ It's active components are glycoalkaloids, glycoproteins, polysaccharides and polyphenolic compounds such as gallic acid, catechin, protocatechuic acid, caffeic acid, epicatechin, rutin and naringenin. $^{12}$ Glycoprotiens are the main effective compound of this plant. Antioxidant potential of isolated glycoprotein has been evaluated by several methods like DPPH, superoxide radical and hydroxyl radical assay. From these results it has been suggested that glycoprotein of $S$. nigrum plant, has potent anti-oxidative potential. ${ }^{13}$ Many studies showed the biochemical evidence of nephroprotective and nephrocurative property of this plant. This study was done to evaluate the histological evidence of the nephroprotective as well as nephrocurative property of S. nigrum.

\section{Plant morphology}

S. nigrum plant can be identified by some of the following key features. It is $25-100 \mathrm{~cm}$ tall, erect annual herb with slightly hairy leaves. Its small star-shaped flowers are white (often tinged with purple) and borne in small clusters in the leaf forks. Stems are often angular, sparsely-pubescent. Its fruit are globose, 8-10 mm in diameter, dull green when young, turning purplish-black and remaining dull in nature as they mature.

\section{METHODS}

This study was conducted in Department of Pharmacology, L.L.R.M. Medical College, Meerut, UP, India from January 2015 to October 2015. Total duration of study was ten months. Approval was taken from institutional animal ethical committee of Lala Lajpat Rai Medical College, Meerut, India, registered under CPCSEA India (Registration No. 819/04/ac/CPCSEA). Albino rats weighed about $200 \mathrm{gm}$ were obtained from the central animal house of the institute. The rats were put in cages under controlled temperature $\left(25^{\circ} \mathrm{C}\right)$ and alternating periods of light and darkness of 12 hours each. They were allowed for free access to standard rat pellet diet and tap water ad libitum. The rats were considered fit for study after 7 days of acclimatization. Gentamicin manufactured by Abott Healthcare Pvt. Ltd (Gentycin) was used to induce gentamicin nephrotoxicity. Aqueous extract of dried fruits of S. nigrum was used as a test drug.

\section{Plant material}

S. nigrum dry fruits were procured from the local market of Meerut, Uttar Pradesh, India. The dry fruits were identified and authenticated by the scientists in Botany Department of Chaudhary Charan Singh University, Meerut, (UP) India.

\section{Preparation of the dry fruit extract}

The dry fruits were separated and powdered with a herb grinder. The dried powder was weighted and transferred to a conical flask containing distilled water and kept in a shaker for 24 hours under air tight condition. After 24 hours it was filtered using Whatmann no.1 filter paper and centrifuged. The supernatant was collected and evaporated till solid yield of the extract was obtained and this extract was used for study. When needed the extract was suspended in water and used in the study. ${ }^{14}$

\section{Study procedure}

Evaluation of nephroprotection was done for three periods of 10,20, 30 days and based on duration, the groups were named G10, G20, G30. Rats were randomized in three groups of 18 rats each. Rats of each main groups were randomized to any of the three subgroups i.e., control (C), gentamicin treated (GT), $S$. nigrum treated (SNT) groups of six rats each.

\section{Group C}

It was the control group and normal saline $(2 \mathrm{ml} / 100$ $\mathrm{gm} /$ day) was given orally once a day, every day for test duration.

\section{Group GT}

In this group, normal saline $(2 \mathrm{ml} / 100 \mathrm{gm} /$ day $)$ was given orally once a day consecutively for test duration and injection gentamicin $(40 \mathrm{mg} / \mathrm{kg})$ was injected intraperitoneally once daily for last five days.

\section{Group SNT}

This group received $S$. nigrum orally (200 mg/kg/day) as a single dose in morning before giving feed for the test duration and, injection gentamicin (40 mg/kg) was injected intraperitoneally once daily for last five days. 
Rats were sacrificed 24 hours after the last dose of gentamicin injection (on $11^{\text {th }}, 21^{\text {st }}$ and $31^{\text {st }}$ day).

The test compounds were administered by the oral gavage method. Rats were fasted three to four hours prior to and one hour after administration. On the last day of study, rats of all groups were kept on fasting for 24 hours (tap water remained freely available) after which they were sacrificed under ketamine $(75 \mathrm{mg} / \mathrm{kg})$ and xylazine $(10 \mathrm{mg} / \mathrm{kg})$ anaesthesia given intra-peritoneally. ${ }^{15}$

The kidneys were excised from the animal and washed with the ice-cold saline. Kidneys were wiped with tissue paper and weighted immediately by electric balance analyzer. Then kidneys were placed in 10\% neutral formalin for 12-24 hours. They were dehydrated and cleared with ethanol and xylene, respectively; followed by embedding in paraffin wax from which blocks were prepared. Sections of $5 \mu \mathrm{m}$ thickness were prepared from the blocks using a microtome. ${ }^{16}$

These were processed in alcohol xylene series and were stained with Harris haematoxylin and eosin stain and subjected to histopathological examination.

Histopathological examination included observation of the following features as glomerular congestion, peritubular congestion, epithelial desquamation, blood vessels congestion, interstitial edema, inflammatory cells, tubular casts and necrosis. Histological changes in kidney was seen under light microscope and gentamicin induced toxicity were graded according to system adopted by Houghton et al. ${ }^{17,18}$

Grade 0: Normal (-).

Grade 1: Tubular damage involving $<25 \%$ of cortical tubules (+).

Table 1: Histopathological changes seen in slides of kidney stained by haematoxylin and eosin at 10, 20 and 30 days duration.

\begin{tabular}{|c|c|c|c|c|c|c|c|c|c|}
\hline \multirow{2}{*}{ Histopathological features } & \multicolumn{3}{|c|}{ G-10 } & \multicolumn{3}{|c|}{ G-20 } & \multicolumn{3}{|c|}{ G-30 } \\
\hline & $\mathbf{C}$ & GT & SNT & $\mathbf{C}$ & GT & SNT & $\mathbf{C}$ & GT & SNT \\
\hline Glomerular congestion & - & ++ & + & - & + & - & - & +++ & - \\
\hline Peritubular congestion & - & ++ & + & - & ++ & + & - & ++ & + \\
\hline Epithelial desquamation & - & + & + & - & + & + & - & + & + \\
\hline Blood vessel congestion & - & +++ & ++ & - & +++ & + & - & ++ & - \\
\hline Interstitial oedema & - & +++ & ++ & - & ++ & + & - & ++ & + \\
\hline Inflammatory cells & - & +++ & ++ & - & ++ & + & - & + & + \\
\hline Tubular casts & - & + & + & - & + & - & - & ++ & - \\
\hline Necrosis & - & + & - & - & + & - & - & + & - \\
\hline
\end{tabular}

The renal interistitium demonstrated infiltration of polymorphoneuclear leukocytes, lymphocytes, plasma cells and macrophages particularly around the necrotic tubules. The renal vascular changes were limited to focal area of congestion.
Grade 2: Tubular damage involving $25 \%$ and $<50 \%$ of cortical tubules $(++)$

Grade 3: Tubular damage involving $50 \%$ and $<75 \%$ of cortical tubules $(+++)$.

Grade 4: Tubular damage involving $75 \%$ of cortical tubules $(++++)$.

\section{Statistical analysis}

Mean \pm SE was calculated for each group. Normality of the data was assessed using Kolmogorov-Smirnov test. Statistical analysis was done using t-test for normally distributed data and using Mann-Whitney test for nonnormal data. P-values $<0.05$ were considered as significant. $\mathrm{P}$ values were estimated by referring to appropriate tables.

\section{RESULTS}

\section{Histopathological changes}

All slides were examined under light microscope. GT group revealed morphological change mainly involving the renal tubules showing patchy necrosis along with hyaline and granular casts in their lumina. Dropped out tubular cells were also seen in the lumina of some of the renal tubules. The lining epithelial cells exhibited hydropic changes with cytoplasmic vacuoles in some areas while regeneration in others. The regenerating cells were recognized by enlarged nuclei. The changes in glomeruli were not very marked. The glomeruli showed mild hypercellularity and congestion in most of the sections. 
the three groups. Regenerating cells were densely populated in the slides of 20 and 30 days group. The histological changes were graded as mentioned in Table
1. Table 2 shows kidney weights in grams in C, GT and SNT groups.

Table 2: Weight of kidneys in C, GT and SNT groups at different test durations (n=6).

\begin{tabular}{|llll|}
\hline $\begin{array}{l}\text { Duration of treatment } \\
\text { (days) }\end{array}$ & $\begin{array}{l}\text { Kidney weight in C group } \\
\text { (mean } \pm \text { SE) (in gms) }\end{array}$ & $\begin{array}{l}\text { Kidney weight in GT } \\
\text { group (mean } \pm \text { SE) } \\
\text { (in gms) }\end{array}$ & $\begin{array}{l}\text { Kidney weight in SNT } \\
\text { group (mean } \pm \text { SE) } \\
\text { (in gms) }\end{array}$ \\
\hline $\mathbf{1 0}$ & $0.59 \pm 0.02$ & $0.99 \pm 0.11^{\alpha}$ & $0.82 \pm 0.06^{\varepsilon \lambda}$ \\
\hline $\mathbf{2 0}$ & $0.6 \pm 0.02$ & $1.01 \pm 0.06^{\beta}$ & $0.60 \pm 0.02^{\zeta \mu}$ \\
\hline $\mathbf{3 0}$ & $0.59 \pm 0.02$ & $1.03 \pm 0.11^{\gamma}$ & $0.6 \pm 0.02^{\eta v}$ \\
\hline
\end{tabular}

${ }^{\alpha} \mathrm{p}<0.05$ as compared to 10 days of control, ${ }^{\beta} \mathrm{p}<0.05$ as compared to 20 days of control, ${ }^{\gamma} \mathrm{p}<0.05$ as compared to 30 days of control, ${ }^{\varepsilon} \mathrm{p}<0.05$ as compared to 10 days of control, ${ }^{\zeta} p>0.05$ as compared to 20 days of control, ${ }^{\eta} p>0.05$ as compared to 30 days of control, ${ }^{\lambda} \mathrm{p}<0.05$ as compared to 10 days of GT, ${ }^{\mu} \mathrm{p}<0.05$ as compared to 20 days of GT and ${ }^{v} \mathrm{p}<0.05$ as compared to 30 days of GT.

\section{DISCUSSION}

Drug induced renal injuries constitute approximately 20 percent of community and hospital acquired episodes of acute renal failure. ${ }^{19,20}$ In elderly, the incidence of druginduced nephrotoxicity may be as high as 66 percent. $^{21}$ Aminoglycoside such as neomycin, gentamicin and tobramicin cause nephrotoxicity as their major side effect. The number of patients developing nephrotoxicity increases with duration of therapy reaching $50 \%$ if the therapy is continued for 14 days or more. ${ }^{22}$

\section{After 10 days of study}

In this study the mean kidney weight was increases on day 10 study in both GT and SNT group as compared to control group. This was correlated with the fact that there is instanced inflammation in the epithelium of proximal convoluted tubule (PCT) during this period.

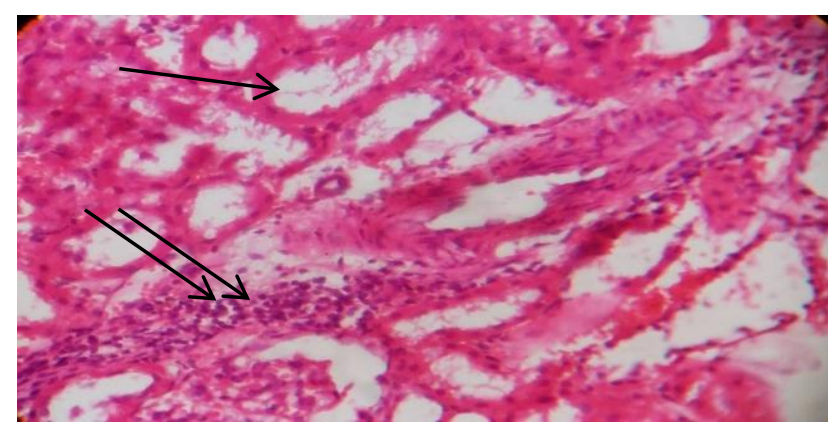

Figure 1: Light micrograph of section from kidneys stained with hematoxylin-eosin (40X). Distorted histological architecture of renal tubules (black arrow) in rats of GT group after 10 days. Numerous mononuclear inflammatory cells (double black arrow) are seen.

Mitochondria became swollen, nuclei of most proximal tubular cells were enlarged and contained prominent central areas of clumped chromatin, lysosomes increases in number with myloid body inside them and cisternae of the rough endoplasmic reticulum were markedly dilated as proven by one of the pioneer study under electron microscope. ${ }^{18}$ This all leads to an inflamed kidney and increase in kidney weight. But in this study mean weight of SNT group was significantly low as compared with GT group. This suggested that $S$. nigrum had antioxidant property so that it can limit the inflammation to some extent. This was also confirmed when histological comparison was done under $10 \mathrm{X}$ and $40 \mathrm{X}$ magnification between two groups at day 10. There was less amount of destruction of PCT in SNT group as compared with GT group (Figures 1 and 2).

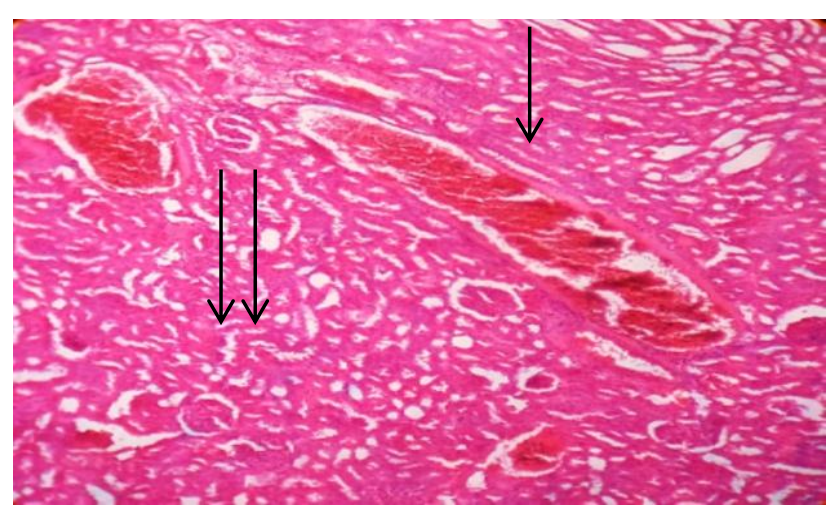

Figure 2: Light micrograph of sections from kidneys stained with hematoxylin-eosin (10X). Blood vessels congestion (black arrow) with normal glomerulus (black arrow) in SNT group after 10 days.

\section{After 20 days of study}

The mean weight of kidney was significantly more in GT group and normal in SNT group as compared to control group. The kidneys have a good potential to compensate for tubular injuries. ${ }^{23}$ Houghton et al found in their study that destruction of PCT epithelium was complete at $14^{\text {th }}$ day, regeneration of epithelium start at $10^{\text {th }}$ day and maturation continue to next three weeks. In their study Distal convoluted tubule (DCT) and cortical convoluted tubules (CCT) also showed structural destruction after 12 days. ${ }^{18}$ Histological examination of the slides under $10 \mathrm{X}$ and 40X magnifications showed dense regenerating cells, recognized by enlarged nuclei. The changes in glomeruli were not very marked (Figures 3 and 4). 


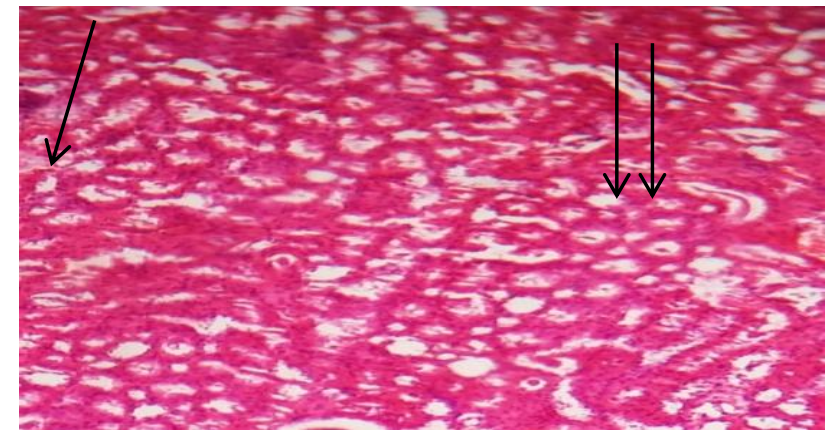

Figure 3: Light micrograph from section from kidney stained with hematoxylin-eosin (40X). Renal architectures in rats of GT group after 20 days. Inflammatory cells, interstitial edema (black arrow) and distorted tubular structure (double arrow).

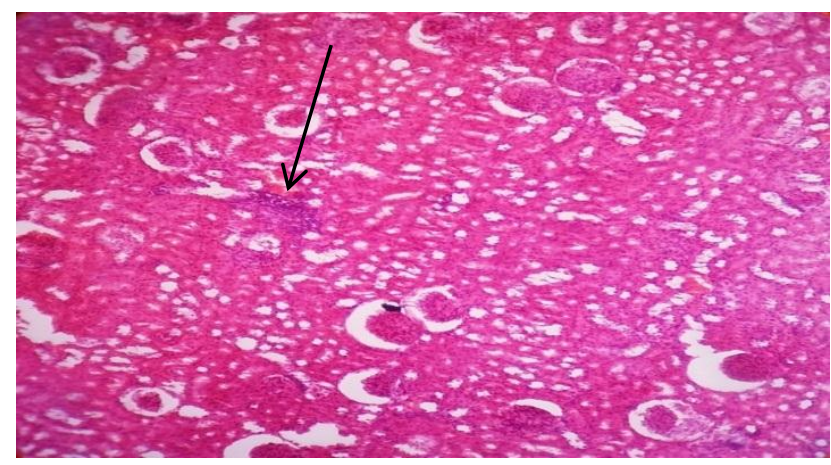

Figure 4: Light micrograph from section from kidney stained with hematoxylin-eosin (10X). Renal architectures in rats of SNT group after 20 days. Inflammatory cells, interstitial edema (black arrow) are seen.

In this study at 20 days duration, the mean weight of kidney in SNT group was significantly low as compared with GT group but not significantly high as compared to control group. This suggest the nephroprotective activity of S. nigrum is time bound and take at least two week for this because there was significant weight gain of kidney at 10 days duration as compared to control group. $S$. nigrum is reported to act as an effective antioxidant of major importance against diseases and degenerative processes caused by oxidative stress. ${ }^{24}$ Thus, the antioxidant property of the extract of these plants may be due to the presence of a high content of polyphenolic compounds such as flavonoids, steroids, vitamin $\mathrm{C}$ and $\beta$ carotene.

\section{After 30 days of study}

The mean weight of kidney was significantly more in GT group and normal in SNT group as compared to control group. In this study at 30 days duration, the mean weight of kidney in SNT group was significantly low as compared with GT group but not significantly high as compared to control group. This result was same as found in 20 days duration results. Mean kidney weight was maximum in GT group among all three groups. Houghton et al in his study found that regenerating and residual cells start growing after 10 days and gradually matured in three weeks to become fully functional as the original cell gentamicin toxicity spares the glomeruli and destroy mostly PCT and if later on DCT and CCT also. ${ }^{18}$ Histologically in the slides under 10x magnification, showed minimal inflammatory cells and near normal tubular structure in SNT group (Figures 5 and 6).

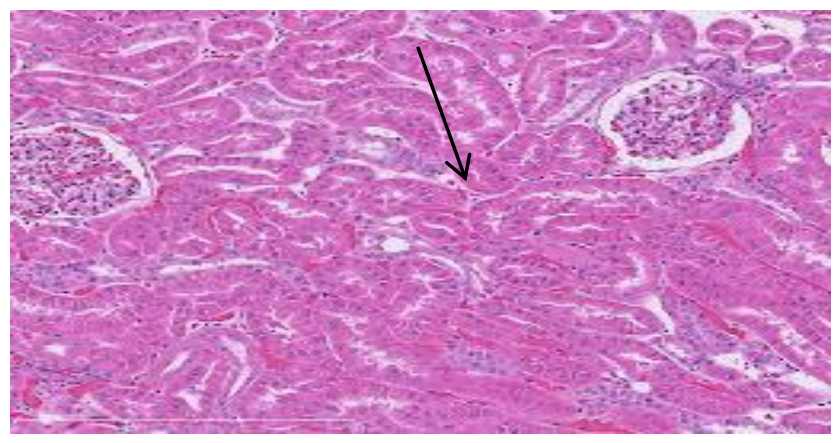

Figure 5: Light micrograph of sections from kidneys stained with hematoxylin-eosin (10X). Restoration of normal architect of renal tubule (black arrow) in SNT group after 30 days.

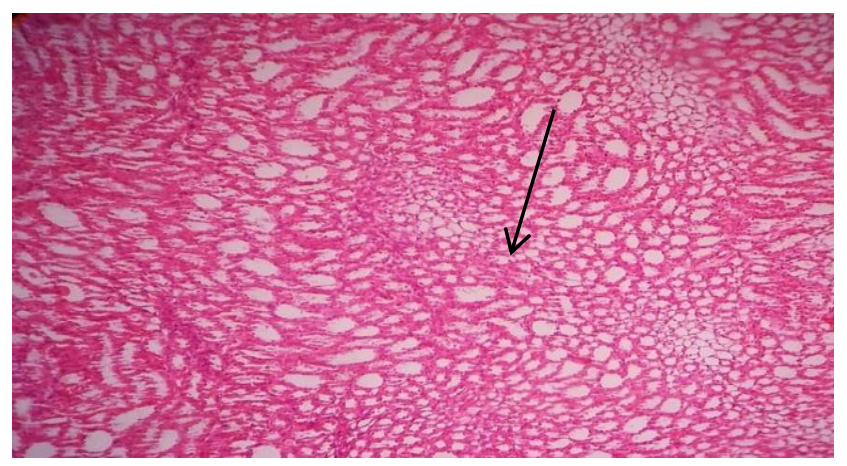

Figure 6: Light micrograph of section from kidney stained with hematoxylin-eosin (10X). Restoration of tubular structure (black arrow) with prominent large nuclei in GT group after 30 days.

Gentamicin toxicity causes permanent destruction of some of the tubule that reflect as fibrosis and scarring of sub-capsular as well as deeper layer in the kidney. This is the cause of suboptimal kidney function even after near normal structural rebuilt of kidney. ${ }^{18}$

\section{CONCLUSION}

The results of this study suggest that the $S$. nigrum fruits extract contains constituents with nephroprotective activities. Based on the observations in the present study S. nigrum extract supplementation may provide a cushion against drug-induced nephrotoxicity. We need more study with increase duration of gentamicin injection to see its action on kidney. 
Funding: No funding sources

Conflict of interest: None declared

Ethical approval: The study was approved by the Institutional Animal Ethics Committee

\section{REFERENCES}

1. Hall JE, Guyton AC. Urine concentration and dilution: regulation of extracellular fuid osmolarity and sodium concentration. In: Grulio WR, ed. Guyton and Hall Textbook of Medical Physiology.12th ed. Philadalphia Saunders: Elsevier; 2011: 348-352.

2. Devasmita C, Ziauddin A. Drug associated renal dysfunction and injury. Nat Clin Pract Nephr. 2006;2(2):80-1.

3. Sakhuja V, Jha V, Ghosh AK. Chronic renal failure in India. Nephrol Dial Transplant. 1994;9:871-2.

4. Modi GK, Jha V. The incidence of end-stage renal disease in India: a population-based study. Kidney Int. 2006;70:2131-3.

5. Bellomo R. The epidemiology of acute renal failure: 1975 versus 2005. Curr Opin Crit Care. 2006;12(6):557-60.

6. Abdel-Zaher AO, Abdel-Hady RA, Mahmoud MM, Farrag MMY. The potential protective role of alphalipoic acid against acetaminophen- induced hepatic and renal damage. Toxicol. 2008;243:261-70.

7. Laskshmi BV, Sudhakar M. Protective effect of Zingiber officinale on gentamicin induced nephrotoxicity in rats. Ind J Pharmacol. 2010;6:5862.

8. Zhang HA, Wang M, Zhou J, Yao QY, Ma JM, Jiang CL. Protective effect of ginsenoside against acute renal failure and expression of tyrosine hydroxylase in the locus coeruleus. Physio Res. 2010;59:61-70.

9. Brinda P, Sridharan G, Pradeep V, Sasikumar S. Antitumor activity and in vivo antioxidant status of hyptissuaveol against Ehrich ascites carcinoma in swiss albino mice. Inves New Drug. 2008;45:10-4.

10. Acharya E, Pokhrel B. Ethno-medicinal plants used by Bantar of Bhaudaha, Morang, Nepal. Our Nature. 2006;4:96-103.

11. Rani P, Khullar N. Antimicrobial evaluation of some medicinal plants for their anti-enteric potential against multidrug resistant Salmonella typhi. Phytother Res. 2004;18:670-73.

12. Ravi V, Saleem TSM, Maiti PP, Gauthaman K, Ramamurthy J. Phytochemical and pharmacological evaluation of Solanum nigrum Linn. Afr J Pharm Pharmacol. 2009;3:454-7.
13. Lim K, Heo H, Lee S. Cytotoxic effect of glycoprotein isolated from Solanum nigrum L, through the inhibition of hydroxyl radical induced DNA-binding activities of NF-kappa B in HT-29 cells. Envi Toxico Pharm. 2004;17:45-54.

14. Hui-Mei L, Hsien-Chun T, Chau-Jong W, Jin-Jin L, Chia-Wen L, Fen-Pi C. Hepatoprotective effects of Solanum nigrum Linn extract against CCl4-iduced oxidative damage in rats. Chem Biol Interact. 2008;171:283-93.

15. Zbigniew K, Przemystaw K, Morys J. General anaesthesia in rats undergoing experiments on the central nervous system. Folia Morphol. 2001;60(4):235-42.

16. Talib VH. A handbook of medical laboratory technology. 2nd ed. New Delhi: CBS Publishers and Distributors; 2007: 155-167.

17. Erdem A, Gundogan M, Usubutun A, Kilinc K, Erdem S, Kara A, et al. The protective effect of taurine against gentamicin-induced acute tubular necrosis in rats. Nephrol Dial Transplant. 2000:117582.

18. Houghton DC, Hartnett M, Cambell-Boswell M, Poeter G, Bennett WA. Light and electron microscopic analysis of gentamicin nephrotoxicity in rats. Am J Path. 1976;82:589-612.

19. Kaufman J, Dhakal M, Patel B, Hamburger R. Community-acquired acute renal failure. Am $\mathrm{J}$ Kidney Dis. 1991;17(2):191-8.

20. Nash K, Hafeez A, Hou S. Hospital-acquired renal insufficiency. Am J Kidney Dis. 2002;39(5):930-6.

21. Kohli HS, Bhaskaran MC, Muthukumar T. Treatment-related acute renal failure in the elderly: a hospital-based prospective study. Nephrol Dial Transplant. 2000;15(2):212-7.

22. Luft FC. Clinical significance of renal changes engendered by aminoglycosides in man. J Antimicrob Chemother. 1984;13(SA):23-30.

23. Barza M, Ioannidis JP, Cappelleri JC, Lau JC. Single or multiple daily doses of aminogycosides :a metaanalysis. Br Med J. 1996;312:338-45.

24. Lin HM, Tseng HC, Wang CJ, Lin JJ, Lo CW, Cho FP. Hepatoprotective effects of Solanum nigrum Linn extract against $\mathrm{CCl} 4$ induced oxidative damage in rats. Chemico Biol Interact. 2008;171:283-93.

Cite this article as: Kushwaha V. Histological evaluation of nephroprotective effect of Solanum nigrum fruit extract against gentamicin induced nephrotoxicity in experimental rats. Int J Basic Clin Pharmacol 2019;8:2191-6. 